\title{
Guest Editorial Special Issue on Advances in Multimedia Computing, Communications and Applications
}

\author{
Paisarn Muneesawang • Feng Wu • Itsuo Kumazawa • \\ Kim-Hui Yap • Hong-Yuan Mark Liao
}

Received: 28 April 2011 /Revised: 2 May 2011 /Accepted: 2 May 2011 / Published online: 4 June 2011

(C) Springer Science+Business Media, LLC 2011

This special issue serves as a forum for recent developments in multimedia signal processing and applications. The ten papers published in this special issue cover a wide range of techniques and applications in various multimedia processing tasks, including multimedia analysis and retrieval, compression and optimization, communication and networking, and multimedia system and applications. In the first paper, Kung and Mak present the paper "PDA-SVM hybrid: a unified model for kernel-based supervised classification.” They have developed a versatile classifier endowed with a broad array of parameters to cope with various kinds of real-world multimedia data.

Two papers in the issue explore techniques in media annotation and tagging. $\mathrm{Lu}$ and Ip present the relevance model-based framework for an automatic image annotation approach and propose a generalized relevance model to solve the problems with the traditional relevance models.

\footnotetext{
P. Muneesawang $(\bowtie)$

Naresuan University,

Phisanulok 65000, Thailand

e-mail: paisarnmu@nu.ac.th

F. Wu

Microsoft Research Asia,

Beijing 100109, People's Republic of China

I. Kumazawa

Tokyo Institute of Technology,

Yokohama 226-8503, Japan

K.-H. Yap

Nanyang Technological University,

Block S2.2, Nanyang Avenue,

Singapore 638798, Singapore

H.-Y. M. Liao

Institute of Information Science, Academia Sinica,

Taipei, Taiwan
}

Sandnes then proposes an automatic method for extracting geo-spatial information from the image contents. The elevation of the sun is first estimated indirectly by measuring the relative length of objects and their shadows in image scenes, and then combined with the creation time of the image for estimating the approximate geographical location of the photographer.

The next three papers investigate visual processing techniques in multimedia research for object detection and tracking as well as for face recognition. Huang and $\mathrm{Li}$ present two new techniques: color-salience weighted probability-of-boundary ( $\mathrm{cPoB}$ ) and successive classification and refinement (SCR) for automatic object detection and tracking in active video. Khan et al. propose a robust visual object tracking scheme that uses the dynamics of object shape and appearance similarity. The method uses a particle filter combined with a multi-mode anisotropic mean shift to perform tracking. Lu et al. investigate the face recognition problem by using a 2-D linear discrimination analysis approach. In this work, they contribute to the optimization of the weighted matrix in the discriminator and also give an iterative algorithm for evaluating the weighting matrix.

Another group of three papers concentrate on multimedia compression and optimization. Lin and Hsu present intraframe transcoding architecture for MPEG-2/H.263/H.264/ MPEG-4 AVC to H.264/MPEG-4 AVC via discrete cosine transform coefficients and coding modes. An I4MB/I16MB mode selection is employed by the summation of the low frequency AC coefficients. An I4MB mode refinement scheme is designed to eliminate the unlikely intra prediction modes for H.263 and H.264/MPEG-4 AVC coding stream. Wang et al. introduce a macro-block group (MBG) parallelism for multicore processors in the AVS coding scheme and compare the coding performance with that of slice-level parallelism. Yeh et al. describe a channel selection algorithm to enhance the 
transmission rate for scalable video coding (SVC) over a multiinput multi-output (MIMO) wireless system in this work. This algorithm allows each layer in SVC to choose its appropriate channel in wireless MIMO systems based on channel state information for transmission rate enhancement.

Liang and Emmanuel address a principled approach to mobile agent code obfuscation. They argue for using a selfmodifying code for obfuscation. This is important since the self-modifying code is usually associated with viruses which are detrimental to security. This work shows how dynamic linking based obfuscation can be enabled by the self-modifying code. A judicious use of junk bytes is made use of to strengthen security.

The quest editors would like to take this opportunity to thank all the authors for their contributions to this special issue, the reviewers for their insightful comments and precious time in reviewing the articles, and the Editor-inChief, Prof. Sun-Yuan Kung, for his valuable advice and strong support in making this special issue possible.

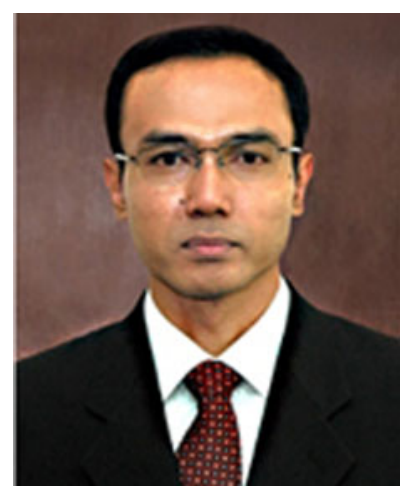

Paisarn Muneesawang received the $\mathrm{PhD}$ degree from the School of Electrical and Information Engineering from the University of Sydney in 2002. In 2003-2004, he held Post-Doctoral Research Fellow position at Ryerson University, Canada. In 2005-2006, he was a faculty member at the College of Information Technology, the University of United Arab Emirates, Al-Ain, UAE. He is currently an Associate Professor and Vice President for Administrative Affairs at Naresuan University, Thailand. His research interests include multimedia signal processing, information system, computer vision, and machine learning. He was an organizing committee member of the 2000 IEEE Pacific-Rim Conference on Multimedia and 2000 IEEE Workshop on Neural Networks for Signal Processing, which were held in Sydney, Australia. He has also co-organized a conference special session on "Moving from Content to Concept Based Image/ Video Retrieval" in 2004 IEEE Pacific-Rim Conference on Multimedia, Tokyo, Japan. In 2004, he co-guest-edited the special issues on "Information Mining from Multimedia Databases" for EURASIP Journal on Applied Signal Processing. He has served as registration Co-Chair for the International conference on multimedia \& expo (ICME'06), and technical program Co-Chair for the 2009 IEEE Pacific-Rim Conference on Multimedia (PCM2009). He is a co-author of two books, Multimedia Database Retrieval: a Human-Centered Approach, published by Springer in 2006, and Advances in Multimedia Information Processing, published by Springer in 2009.

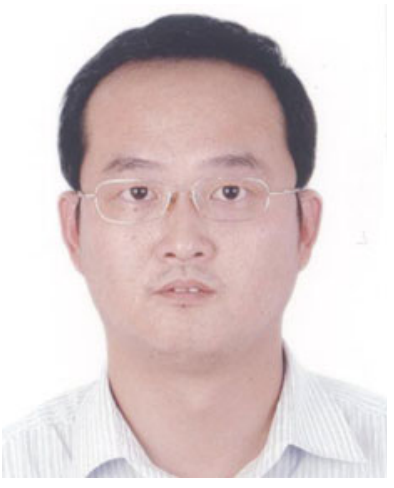

Feng Wu received his B.S. degree in Electrical Engineering from XIDIAN University in 1992. He received his M.S. and Ph.D. degrees in Computer Science from Harbin Institute of Technology in 1996 and 1999, respectively. Wu joined in Microsoft Research Asia, formerly named Microsoft Research China as an Associate Researcher in 1999. He has been a researcher with Microsoft Research Asia since 2001 and is now a Senior Researcher/Research Manager.

His research interests include image and video representation, media compression and communication, as well as, computer vision and graphics. He has authored or co-authored over 200 papers published in journals like IEEE Transaction on Image Processing, IEEE Transactions on Circuits and System for Video Technology, IEEE Transactions on Signal Processing, IEEE Transactions on Visualization and Computer Graphics, IEEE Transactions on Multimedia, IEEE Transactions on Wireless Communication and some other International Conferences and Forums, e.g., MOBICOM, SIGIR, INFOCOM, CVPR, DCC, and ICIP. As a co-author, he got the best paper award in IEEE T-CSVT 2009, PCM 2008 and SPIE VCIP 2007. Wu has been a senior member of IEEE. He serves as an associate editor for IEEE Transaction on Circuits and Systems for Video Technology and International Journal of Multimedia. He also serves as TPC chair in MMSP 2011, VCIP 2010 and PCM 2009, TPC track chair in ICME 2011 and ICME 2009 and Special sessions chair in ICME 2010.

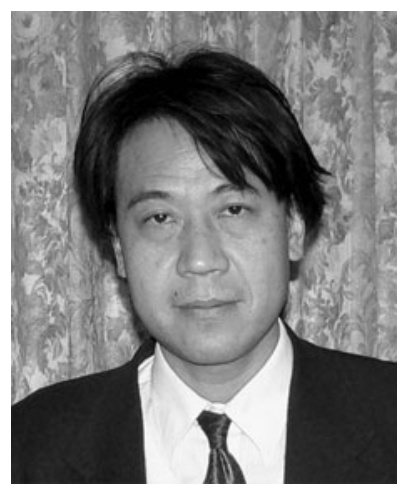

Itsuo Kumazawa received the M.S. and Doctor of Engineering degrees from Tokyo Institute of Technology in 1983 and 1986, respectively. He is a Professor and the director of Imaging Science and Engineering Lab in the Department of Computer Science at Tokyo Institute of Technology. He held visiting professor position at the University of Toronto. He is the recipient of Tejima Memorial Award, Shinohara Memorial Award, and the Institute of Electronics, Information and Communication Engineers Best Paper Award. He was an 
editor of the journals for Information Processing Society of Japan. Dr. Kumazawa has authored/coauthored more than 100 scientific publications in pattern recognition, media understanding, machine learning, computer vision, and information processing.

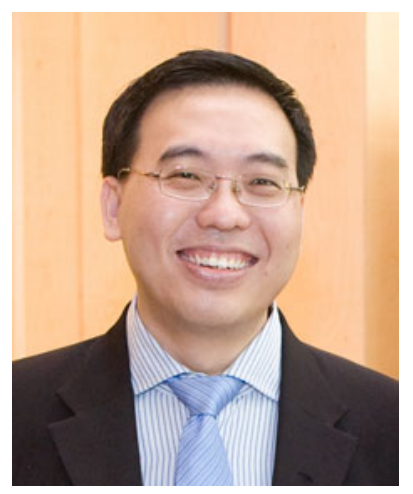

Dr. Kim-Hui Yap received the B. Eng. and Ph.D. degrees in electrical engineering from the University of Sydney, Australia, in 1998 and 2002, respectively. Since then, he has been a faculty member at Nanyang Technological University, Singapore, where he is currently an Associate Professor. His main research interests include image/ video processing, media content analysis, computer vision, and computational intelligence. He has served as an Associate Editor for the IEEE Computational Intelligence Magazine and Journal of Signal Processing Systems. He has also served as an Editorial Board Member for The Open Electrical and Electronic Engineering Journal, and as a Guest Editor for the IEICE Transactions on Fundamentals. Dr. Yap is a Senior Member of IEEE. He has served as the Treasurer of the IEEE Singapore Signal Processing Chapter and the Committee Member of the IEEE Singapore Computational Intelligence Chapter. He has also served as the Finance Chair in 2010 IEEE International Conference on Multimedia \& Expo, the Workshop Co-chair of the 2009 MDM International Workshop on Mobile Media Retrieval, and others. He served as the Group Leader in Content-based Analysis for the Center for Signal Processing, Nanyang Technological University, Singapore. $\mathrm{He}$ has numerous publications in various international journals, book chapters and conference proceedings. He has authored a book entitled "Adaptive Image Processing: A Computational Intelligence Perspective, Second Edition" published by the CRC Press in 2009, and edited a book entitled "Intelligent Multimedia Processing with Soft Computing" published by Springer-Verlag in 2005 .

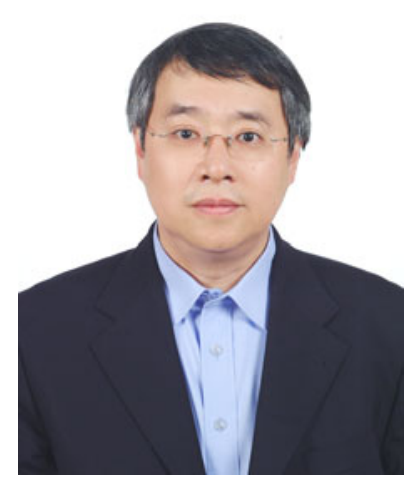

Hong-Yuan Mark Liao received a BS degree in physics from National Tsing-Hua University, Hsin-Chu, Taiwan, in 1981, and an MS and Ph.D degree in electrical engineering from Northwestern University in 1985 and 1990, respectively. In July 1991, he joined the Institute of Information Science, Academia Sinica, Taiwan. He is a Research Fellow now. In 2008, he became the division chair of the computer science and information engineering division II, National Science Council of Taiwan. $\mathrm{He}$ is jointly appointed as a professor of the Computer Science and Information Engineering Department of National Chiao-Tung University. Since Feb. 2009, he has been jointly appointed as the Multimedia Information Chair Professor of National Chung Hsing University. From August 2010, he has been appointed as an Adjunct Chair Professor of Chung Yuan Christian University. His current research interests include multimedia signal processing, video-based Surveillance Systems, video forensics, and multimedia protection.

Dr. Liao was a recipient of the Young Investigators' award from Academia Sinica in 1998. He received the distinguished research award from the National Science Council of Taiwan in 2003 and 2010, and the National Invention Award of Taiwan in 2004. In 2008, he received a Distinguished Scholar Research Project Award from National Science Council of Taiwan. In 2010, he received the Academia Sinica Investigator Award. In June 2004, he served as the conference co-chair of the 5th International Conference on Multimedia and Exposition (ICME) and technical co-chair of the 8th ICME held at Beijing. In Jan. 2011, Dr. Liao served as General co-chair of the 17th International Conference on Multimedia Modeling. From 2006-2008, Dr. Liao was the president of the Image Processing and Pattern Recognition Society of Taiwan.

Dr. Liao is on the editorial boards of the IEEE Signal Processing Magazine, the IEEE Transactions on Image Processing, and the IEEE Transactions on Information Forensics and Security. He was an associate editor of IEEE Transactions on Multimedia during 1998-2001. 